\title{
Quantitative 3D analysis of structural organization of normal and tumor cells
}

\author{
Carolyn A. Larabel1 ${ }^{1,2,3}$ and Mark A. Le Gros ${ }^{1,2,3}$
}

${ }^{1 .}$ Department of Anatomy, University of California San Francisco, San Francisco USA.

2. Molecular Biophysics and Integrated Bioimaging, Lawrence Berkeley National Laboratory, Berkeley USA.

${ }^{3 .}$ National Center for X-ray Tomography, Lawrence Berkeley National Laboratory, Berkeley USA.

Soft X-ray tomograpahy (SXT) is similar in concept to the well-established medical diagnostic technique, computed axial tomography (CAT), except SXT is capable of imaging with a spatial resolution of $50 \mathrm{~nm}$ or better ${ }^{1}$. With SXT we can examine whole, hydrated cells (between 10-15 $\mu \mathrm{m}$ thick), eliminating the need for time-consuming embedding and sectioning procedures. Cells are imaged using X-ray energies between the $\mathrm{K}$ shell absorption edges of carbon $(284 \mathrm{eV}, \lambda=4.4 \mathrm{~nm})$ and oxygen $(543 \mathrm{eV}, \lambda=2.3 \mathrm{~nm})^{2}$. In this energy range, photons readily penetrate the aqueous environment while encountering significant absorption from carbon- and nitrogen-containing organic material. Consequently, organic material absorbs approximately an order of magnitude more strongly than water, producing a quantifiable natural contrast image of cellular structures. SXT, like other tomography modalities, requires recording images from multiple different viewing angles. By collecting images from multiple angles through 360 degrees of rotation, SXT reconstructions yield information at isotropic resolution.

Images are formed using unique optics called zone plates (ZP). An X-ray ZP optic consists of a number of concentric nanostructured metal rings, or zones, formed on a thin X-ray transmissive silicon nitride membrane. The width of the outermost ring determines the spatial resolution of the ZP lens, whereas the thickness of the rings determines the focusing efficiency ${ }^{3}$. In the microscope we utilize, the condenser ZP lens has an overall diameter of $1 \mathrm{~cm}, 41,667$ zones made with approximately $200 \mathrm{~nm}$ thick nickel, and a $5 \mathrm{~mm}$ central stop. The high-resolution objective ZP lens has a diameter of $63 \mu \mathrm{m}$ and an outer zone width of $50 \mathrm{~nm}$ to assure the entire cell is in focus.

Because SXT is fast ( 5 min per tomographic data set), we can examine large numbers of cells. Since organic material absorbs approximately an order of magnitude more strongly than water, the highcontrast image of cellular structures is quantifiable. X-ray absorption follows Beer's Law, therefore the absorption of photons is linear and a function of the biochemical composition at each point in the cell. As a result, a linear absorption coefficient (LAC) value of each voxel can be calculated ${ }^{4}$. For example, lipid drops with high concentrations of carbon are more highly absorbing $\left(\mathrm{LAC}=0.7 \mathrm{~m}^{-1}\right)$ than fluidfilled vesicles $\left(\mathrm{LAC}=0.2 \mathrm{~m}^{-1}\right)$.

To determine the location of specific molecules with respect to cellular structures, we developed high numerical aperture cryogenic fluorescence tomography (CFT) for correlated imaging studies. This multi-modal approach - imaging the same cell using both CFT and SXT - allows localization of genetically encoded fluorescent molecules directly in the context of a high-resolution 3-D tomographic reconstruction of the cell.

We used soft x-ray tomography to examine the structural organization of normal and tumor cells, including cells obtained from mouse tissue, mouse cell lines, and human cells lines. Tumor cells have 
significantly larger nuclei with a decreased percent volume heterochromatin composition. The cell cytoplasm is significantly larger in tumor cells than normal cells and contains greater numbers of mitochondria, endoplasmic reticulum and other organelles.

\section{References:}

[1] CA Larabell and KA Nugent, Curr Op Struct Biol 20(5) (2010), p 623-31.

[2] G McDermott, MA Le Gros, and CA Larabell. 63 (2012), p 225-39.

F Author, S Author and TH Author, Journal volume (year), p. 1.

[3] DT Atwood in Soft X-rays and extreme ultraviolet radiation: Principles and applications, ed. DT Atwood (Cambridge University Press).

[4] The authors acknowledge funding from the National Institute of General Medical Science of the National Institutes of Health, Grant Number P41GM103445, and the US Department of Energy, Office of Biological and Environmental Research (DE-AC02-05CH11231).

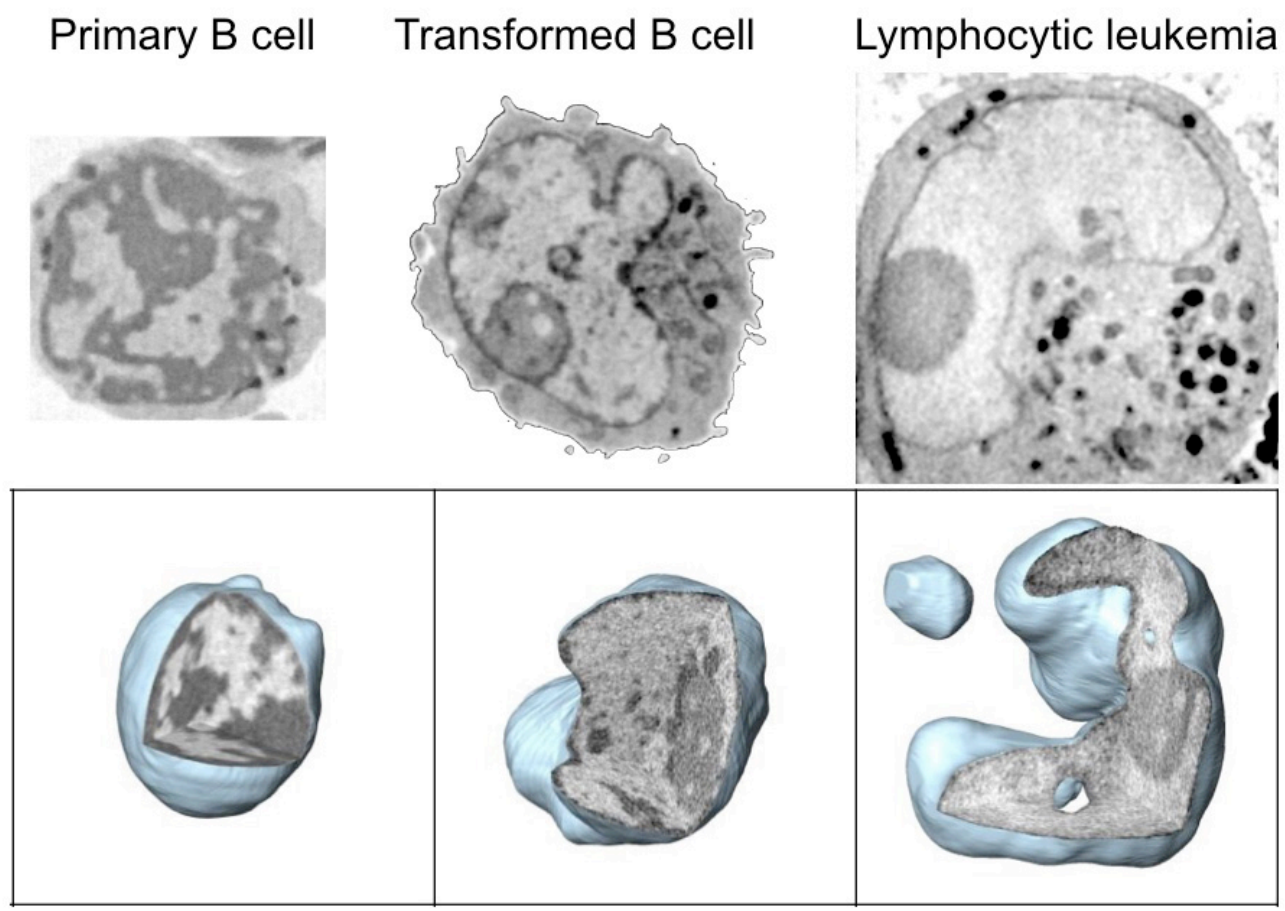

\begin{tabular}{|l|c|c|c|}
\hline & B cell & $\begin{array}{c}\text { Transformed B } \\
\text { cell }\end{array}$ & $\begin{array}{c}\text { Lymphocytic } \\
\text { leukemia }\end{array}$ \\
\hline Nuclear volume & $102 \mu \mathrm{m}^{3}$ & $360 \mu \mathrm{m}^{3}$ & $450 \mu \mathrm{m}^{3}$ \\
\hline$\%$ Vol. heterochromatin & $52 \%$ & $34 \%$ & $22 \%$ \\
\hline Mitochondrial volume & $5.5 \mu \mathrm{m}^{3}$ & $31.5 \mu \mathrm{m}^{3}$ & $26.2 \mu \mathrm{m}^{3}$ \\
\hline
\end{tabular}

Figure 1. Soft x-ray tomography analysis of normal and tumor cells. Top row, orthoslices (virtual sections) through the cells. Middle row, 3D cutaway views of three orthogonal orthoslices. Bottom, quantitative analysis of changes in nuclear volume, percent volume heterochromatin, and mitochondrial volume. 